Physics Vol. 1, No. 1, pp. 49-61, 1964. Pergamon Press, Inc. Printed in the United States.

\title{
QUANTUM MECHANICAL PHASE AND TIME OPERATOR
}

\author{
LEONARD SUSSKIND* \\ Laboratory of Nuclear Studies, Cornell University, Ithaca, New York \\ and \\ JONATHAN GLOGOWER
}

(Received 13 May 1964)

\begin{abstract}
The phase operator for an oscillator is shown not to exist. It is replaced by a pair of non-commuting sin and cos operators which can be used to define uncertainty relations for phase and number. The relation between phase and angle operators is carefully discussed. The possibility of using a phase variable as a quantum clock is demonstrated and the states for which the clock is most accurate are constructed.
\end{abstract}

\section{Introduction}

We shall discuss quantities which are special cases of time operators, that is, variables conjugate to the energy. Bohm and Aharanov ${ }^{1}$ have attempted to use such operators in their discussion of the energytime uncertainty principle. They suppose that an operator exists which is an appropriate quantum variable describing the generalized clock. In fact, Bohm and Aharanov assert that such operators exist for all quantum systems. For example, they consider a clock composed of a single free particle, whose position measures the time. The suggested time operator is

$$
t_{\mathrm{c}}=\frac{1}{2} M\left(X P_{\mathrm{x}}^{-1}+P_{\mathrm{x}}^{-1} X\right)
$$

The questions of existence, Hermiticity, eigenvectors, etc., were not considered.

What we shall do is consider an example of a generalized time operator, the phase of a quantum oscillator, and give answers to these questions for the system of an oscillator clock.

Another reason to study the phase of the quantum oscillator is its possible relevance to the quantum theory of coherence.

The following convention is used. A caret will be used above a quantity to denote an operator. A quantity without a caret is a $c$ number. Also $\hat{\phi}$ will be used as an operator with $\theta$ its eigen-values.

\section{Definition of Phase Variables}

We are going to study the quantum oscillator described by the Hamiltonian

$$
\hat{H}=\hat{P}^{2}+\frac{1}{4} w^{2} \hat{X}^{2}
$$

with special emphasis on the time phase variable.

*National Science Foundation Predoctoral Fellow. 
Classically the solution to this Hamiltonian is the well-known

$$
\begin{aligned}
& X=A e^{i \phi}+A^{*} e^{-i \phi}, \quad \phi=w t \\
& P=i / 2\left[A w e^{i \phi}-A^{*} w e^{-i \phi}\right]
\end{aligned}
$$

The $A$ 's may be chosen real

$$
\begin{aligned}
& X=2 A \cos \phi \\
& P=-A w \sin \phi
\end{aligned}
$$

In quantum mechanics the equivalent equations are

$$
\begin{aligned}
& \hat{X}=\hat{a}^{+}+\hat{a}^{-} \\
& \hat{P}=-i / 2 w\left(\hat{a}^{+}-\hat{a}^{-}\right)
\end{aligned}
$$

where we think of $\hat{a}^{+}$and $\hat{a}$ as the positive and negative frequency components of $\hat{x}$. In analogy with (2) and $\left(2^{\prime}\right)$ we will consider $a^{+}$to be the product of 2 operators, one Hermitian representing the amplitude of oscillation and one complex representing the phase. Ideally we should like to be able to express $\hat{a}^{+}$and $\hat{a}^{-}$ in the form $\hat{R} e^{+i \hat{\phi}}$ and $e^{-i} \hat{\phi} R$ where $R$ is Hermitian and $e^{i \hat{\phi}}$ is a unitary operator defining a Hermitian $\hat{\phi}$. This is what Heitler [2] and Dirac [3] try to do. We shall find, however, that their arguments are not correct. Since the number operator, $n$, is given by $\hat{a}^{+} \hat{a}^{-}=\hat{R} e^{-i \hat{\phi}} e^{i \hat{\phi}} \hat{R}$, where $e^{i \hat{\phi}}$ is unitary, then

$$
\hat{n}=\hat{R}^{2}
$$

Suppose $R$ is Hermitian. Then its representation in some basis is

$$
\hat{R}=\left(\begin{array}{cccc}
R_{1} & & & \\
& R_{2} & & \\
& & \cdot & \\
& & \cdot & \\
& & & \cdot
\end{array}\right) \quad \hat{R}^{2}=\left(\begin{array}{llll}
R_{1}{ }^{2} & & & \\
& R_{2}{ }^{2} & & \\
& & \cdot & \\
& & & \cdot \\
& & & \cdot
\end{array}\right)
$$

But $n$ is diagonal in but one basis system. Hence the system in which $R$ is diagonal is the number representation and therefore

$$
\hat{R}=\sqrt{\hat{n}}, \quad \hat{a}^{+}=\sqrt{\hat{n}} e^{-i \hat{\phi}}, \quad \hat{a}^{-}=e^{i \hat{\phi} \sqrt{\hat{n}}}
$$

These equations can be used to obtain

$$
\left[\hat{n}, \mathrm{e}^{i \hat{\phi}}\right]=\mathrm{e}^{i \hat{\phi}}
$$

This plus the supposed unitarity of $e^{i \hat{\phi}}$ is used to obtain

$$
[\hat{n}, \hat{\phi}]=-i, \quad \hat{\dot{\phi}}=-\omega
$$

However, taking matrix elements of this commutation relation we get

$$
\left(n_{1}-n_{2}\right)<n_{1}|\hat{\phi}| n_{2}>=-i \delta n_{1} n_{2}
$$

This is obviously impossible since when $n_{1}=n_{2}$ the left side is zero while the right side is -1 . The difficulty is not a basic one and lies in not properly taking account of the periodic nature of $\hat{\phi}$. This will be
discussed in detail with angle operators. 
A more important objection is that $e^{i \hat{\phi}}$ defined above is not unitary and does not define an Hermitian $\hat{\phi}$. Consider

$$
\hat{a}^{+}=\sqrt{\hat{n}} e^{-i \hat{\phi}}
$$

Taking matrix elements in the $n$ representation

$$
\delta_{n, m+1} \sqrt{m+1}=\sqrt{n}<n\left|e^{-i \hat{\phi}}\right| m>.
$$

For $n=0$ both sides are identically zero. For $n \neq 0$ we get $\left\langle n\left|e^{-i \hat{\phi}}\right| m\right\rangle=\delta_{n, m+1}$. The arbitrariness of $<0\left|e^{-i \hat{\phi}}\right| m>$ is due to $n$ having zero eigenvalues and therefore no inverse.

Let

$$
<0\left|e^{-i \hat{\phi}}\right| m>=r_{m}
$$

Then

$$
\begin{aligned}
& e^{-i \hat{\phi}}=\left(\begin{array}{cccc}
r_{0} & r_{1} & r_{2} & \ldots \\
1 & 0 & 0 & \ldots \\
0 & 1 & 0 & \ldots \\
0 & 0 & 1 & \ldots \\
. & . & . & \ldots
\end{array}\right) \quad e^{i \hat{\phi}}=\left(\begin{array}{ccccc}
r_{0}{ }^{*} & 1 & 0 & 0 & \ldots \\
r_{1}{ }^{*} & 0 & 1 & 0 & \ldots \\
r_{2}{ }^{*} & 0 & 0 & 1 & \ldots \\
. & . & . & . & \ldots
\end{array}\right) \\
& e^{-i \hat{\phi}_{e}^{i \hat{\phi}}}=\left(\begin{array}{cccc}
\Sigma r_{i} r_{i}^{*} & r_{0} & r_{1} & \cdots \\
r_{0}^{*} & 1 & 0 & \cdots \\
r_{1}^{*} & 0 & 1 & \cdots \\
\cdot & . & . & \cdots
\end{array}\right)
\end{aligned}
$$

This cannot equal unity for any choice of the $r_{i}$. Therefore we shall cease to denote these operators by $e^{i \hat{\phi}}$ but rather call them $\hat{e}^{i \phi}$, to indicate that $\phi$ is not an operator. We can restrict the choice of $r_{i}$ by demanding $\hat{e}^{i \phi} \hat{e}^{-i \phi}=1$ which gives $r_{i}=0$. In fact by so restricting $r_{i}$ we can replace our definition of $\hat{e}^{i \phi}$ by

$$
\hat{e}^{-i \phi}=\hat{a}^{+}(\hat{n}+1)^{-1 / 2}, \quad \hat{e}^{i \phi}=(\hat{n}+1)^{-1 / 2} \hat{a}^{-}
$$

This definition gives the same operators as the previous one. In fact $\hat{e}^{\ell \phi}$ is just the lowering operator

$$
\hat{e}^{i \phi}|m\rangle=|m-1\rangle, \quad \hat{e}^{-i \phi}|m\rangle=|m+1\rangle
$$

It must be kept in mind that $\hat{e}^{i \phi}$ and $\hat{e}^{-i \phi}$ are symbolic expressions and do not represent some exponential function of a Hermitian phase. We can use these operators, however, to define trigonometric functions of phase and investigate their properties especially in the classical limit. We shall show that these operators are observables and that they do become the classical functions of phase in the limit of large amplitudes. Furthermore, they provide a set of quantities for measuring time. Consider

$$
\begin{aligned}
& \cos \hat{\phi} \equiv 1 / 2\left[\hat{e}^{i \phi}+\hat{e}^{-i \phi}\right] \\
& \sin \hat{\phi} \equiv \frac{1}{2 i}\left[\hat{e}^{i \phi}-\hat{e}^{-i \phi}\right]
\end{aligned}
$$

Clearly they are Hermitian operators. According to the principles of quantum theory they are observable dynamical variables if they possess a complete set of eigenvectors. Therefore we shall proceed to investigate their eigenvalue spectrum and eigenvectors. 
Since

$$
[\cos \hat{\phi}, \sin \hat{\phi}]=\left(\begin{array}{ccccc}
1 & 0 & 0 & 0 & . \\
0 & 0 & 0 & 0 & . \\
0 & 0 & 0 & 0 & . \\
. & \cdot & . & . & .
\end{array}\right) \neq 0
$$

the eigenstates of $\cos \hat{\phi}$ and $\sin \hat{\phi}$ will be distinct. That $[\cos \phi, \sin \phi] \neq 0$ is not too surprising since one is essentially the time derivative of the other. In fact, it is the non-commuting nature of $\cos \phi$ and $\sin \phi$ which makes $\hat{e}^{i \phi}$ not unitary.

The non-commuting of $\cos \hat{\phi}$ and $\sin \hat{\phi}$ prevents us from constructing states of known $\phi$. In fact, we shall show that eigenstates of $\cos \hat{\phi}$ can be considered superpositions of states with $\pm \phi$, and $\sin \phi$ states are superpositions of states with $\phi$ and $\pi-\phi$.

We now find the eigenvalues and eigenvectors of $\cos \hat{\phi}$.

Let $|\cos \theta\rangle=\sum_{n} C_{n}|n\rangle$. Remembering that $\hat{e}^{i \phi}$ is a lowering operator and $\hat{e}^{-i \phi}$ a raising operator we obtain

$$
2 \cos \hat{\phi}\left|\cos \theta>=\sum_{n}\left[C_{n}\left|n+1>+C_{n}\right| n-1>\right]=2 \lambda \sum_{n} C_{n}\right| n>
$$

for the eigenvalue equation. Hence

$$
C_{1}=2 \lambda C_{0}, \quad C_{n}+C_{n+2}=2 \lambda C_{n+1}
$$

Apart from an over all multiplicative factor this recursion relation has only one solution for each value of $\lambda$. The general solution to the second equation is

$$
C_{n}=A p^{n}+B p^{-n}
$$

where $p+1 / p=2 \lambda$. Then if $\mid \cos \theta>$ is to be a normalizable vector $|p|$ must equal 1 . Let $p=e^{i \theta}$. Then
$\lambda=\cos \theta$ and we find that $\cos \phi$ has the eigenvalue $\lambda=\cos \theta$ and we find that $\cos \phi$ has the eigenvalue spectrum -1 to +1 . Inserting this into equation (16) we
get

$$
C_{n}=\sin (n+1) \theta
$$

The eigenstate of $\cos \hat{\phi}$ with eigenvalue $\cos \theta$ is

$$
\sum_{n} \sin (n+1) \theta \mid n>
$$

Notice that for each value of $\cos \theta$ there is only one state and not two.

Now $\cos \theta$ is a superposition of $\sum_{n} \mathrm{e}^{i n \theta}|n\rangle$ and $\sum_{n} \mathrm{e}^{-i n \theta} \mid n>$. These states are the states that one might think should be the eigenstates of the conjugate to the $\hat{n}$ operator in analogy with the usual situation for an operator and its conjugate. Thus it might be suspected that $\sum_{n} e^{i n \theta} \mid n>$ provides a good definition of the eigenstates of $\phi$. Might we have done much better in our definitions of phase by considering an operator $\phi$ which multiplies each state $\sum_{n} \mathrm{e}^{i n \theta} \mid n>$ by $\theta$ ? 
For such an operator to be an observable at all, the states

$$
\left.\left|\theta>=\sum_{n} e^{i n \theta}\right| n\right\rangle
$$

must form an orthonormal complete set. Consider the inner product

$$
<\theta\left|\theta^{\prime}\right\rangle=\sum_{0}^{\infty} \mathrm{e}^{i n\left(\theta^{\prime}-\theta\right)}
$$

Were the sum from $-\infty$ to $+\infty$ this would equal $\delta\left(\theta^{\prime}-\theta\right)$. But as it stands it is not the delta function and the states are not orthogonal [4]. Thus if the operator exists at all it is not Hermitian. Furthermore the superposition of states

$$
\int d \theta e^{i m \theta} \sum_{n} e^{i n \theta} \mid n>=0
$$

if $m$ is a positive integer. The states are not even all linearly independent. Hence $\phi$ was not defined in a consistent manner.

Before going on we shall directly prove the orthogonality and completeness of the $\mid \cos \theta>$

$$
\begin{aligned}
<\cos \theta \mid \cos \theta^{\prime}>=\sum_{n} & \sin (n+1) \theta \sin (n+1) \theta^{\prime} \\
= & \sum_{n}\left\{\cos (n+1)\left(\theta-\theta^{\prime}\right)-\cos (n+1)\left(\theta+\theta^{\prime}\right)\right\}=\delta\left(\theta-\theta^{\prime}\right)-\delta\left(\theta+\theta^{\prime}\right)
\end{aligned}
$$

Now we have found that for each value of $\theta$ there exists only one state. That is $|\cos \theta\rangle=-|\cos (-\theta)\rangle$. We can put the $\cos \phi$ states in 1-to- 1 correspondence with the angles only if we restrict $\theta$ to half its range, say from 0 to $\pi$. Then one of the delta functions is spurious. The states $|\cos \theta\rangle$ are then orthogonal.

The remaining question is whether the $\cos \theta$ states are complete. If we can expand an arbitrary $|n\rangle$ state in $\mid \cos \theta>$ states then the $\mid \cos \theta>$ states are indeed complete. Hence we try

$$
\left|n>=\sum_{n} \int_{0}^{\pi} f(\theta) \sin (m+1) \theta\right| m>
$$

Let $f(\theta)=\frac{2}{\pi} \sin (n+1) \theta$. Then

$$
\sum_{m} \int_{0}^{\pi}[\sin (m+1) \theta][\sin (n+1) \theta]|m\rangle=\frac{\pi}{2} \sum_{m} \delta_{n m}|m\rangle=\frac{\pi}{2}|n\rangle
$$

Summarizing the results for the $\cos \hat{\phi}$ operator, it is an observable with spectrum -1 to 1 and the eigenvalues are nondegenerate. Each $\mid \cos \theta>$ state can be thought of as a superposition of $\mid+\theta>$ and $\mid-\theta>$ states. The sense in which the $|\theta\rangle$ states can be thought of as states of known phase will be described later.

Similar results can be obtained for the $\sin \hat{\phi}$ operator. The recursion relation for the eigenstates becomes

$$
C_{m}-C_{m+2}=2 i \mu C_{m+1}, \quad C_{1}=-2 i \mu C_{0}
$$


where $C_{m}=\langle m \mid \sin \theta\rangle$ where $\mu$ is the eigenvalue of $\sin \hat{\phi}$.

We find the non-increasing solutions to be

$$
\left|\sin \theta>=\sum_{m}\left\{e^{-i(m+1) \theta}-e^{-i(m+1)(\pi-\theta)}\right\}\right| m>
$$

Hence the state $|\sin \theta\rangle$ is the same as $|\sin (\pi-\theta)\rangle$.

To put these states into 1 - to- 1 correspondence with angles we use only those angles in the first and fourth quadrant. The proof that $\left\langle\sin \theta \mid \sin \theta^{\prime}\right\rangle=\delta\left(\theta-\theta^{\prime}\right)$ and the completeness of the $\mid \sin \theta>$ states follows in much the same way as the corresponding proof for the cosines.

Thus the observables $\sin \hat{\phi}$ and $\cos \hat{\phi}$ exist but don't commute or define an operator $\hat{\phi}$. In particular the equation $\sin ^{2} \hat{\phi}+\cos ^{2} \hat{\phi}=1$ must be replaced by

$$
\sin ^{2} \hat{\phi}+\cos ^{2} \hat{\phi}=1-i[\cos \hat{\phi}, \sin \hat{\phi}]
$$

We would like to show that $<\cos \theta \mid \sin \theta^{\prime}>$ is large only if $\sin ^{2} \theta^{\prime}+\cos ^{2} \theta=1$. It turns out that

$$
<\cos \theta \mid \sin \theta^{\prime}>=f\left(\theta-\theta^{\prime}\right)-f\left(\theta+\theta^{\prime}\right)+f\left(\theta-\theta^{\prime}-\pi\right)-f\left(\theta+\theta^{\prime}-\pi\right)
$$

where $f(\theta)=\Sigma_{n} \mathrm{e}^{i n \theta}$ which is very sharply peaked at $\theta=0 .{ }^{4}$ Thus if $\theta$ is in the first quadrant the function is peaked at $\theta^{\prime}=+\theta$. If $\theta$ is in the second quadrant the function is peaked at $\theta^{\prime}=\theta-\pi$ and $\pi-\theta$. As expected $<\cos \theta \mid \sin \theta^{\prime}>$ is peaked at $\sin ^{2} \theta^{\prime}+\cos ^{2} \theta=1$.

\section{The Oscillator Clock}

Since $[\cos \hat{\phi}, \sin \hat{\phi}] \neq 0$ and $\sin \hat{\phi}$ is essentially the time derivative of $\cos \hat{\phi}$ we can expect spreading of the wave packet in $\cos \theta$. That is to say if we prepare a state $\mid \cos \theta>$ at time $t=0$, at time $t=\frac{2 n_{\pi}}{\omega}$ the state is not $|\cos (\theta \pm \omega t)\rangle$ but some wave packet in cos space. If we define an operator $\cos (\hat{\phi}+\epsilon)$ by $2 \cos (\hat{\phi}+\epsilon)=e^{i \epsilon} \hat{e}^{i \phi}+e^{-i \epsilon} \hat{e}^{-i \phi}$ which incidentally does not commute with $\cos \hat{\phi}$, we would find the system in an eigenstate of $\cos (\hat{\phi}-\omega t)$ at time $t$. In this sense there is no spreading. However for any fixed choice of $\epsilon$, wave packets in cos space spread with time. We might think that this spreading would prevent us from using the oscillator as a clock since a clock parameter must increase in time with essentially no spread in the possible values of the time which is measured. However, we shall show later that the oscillator can be used as a clock which can measure time with arbitrary accuracy if it is pre-
pared in the correct

We have not yet exhibited states with a small uncertainty in both $\cos \hat{\phi}$ and $\sin \hat{\phi}$ simultaneously. The existence of such states is crucial for the existence of a classical limit. It might be questioned whether such states do exist since every $\mid \cos \theta>$ state is a superposition of $\mid \sin \theta>$ and $|\sin -\theta\rangle$ states. We must show that for every angle $\theta$ there exist states such that $\langle\cos \hat{\phi}\rangle=\cos \theta$ and $\langle\sin \hat{\phi}\rangle=\sin \theta$. to have the desired properties. Consider first the inner product

$$
\begin{aligned}
\left\langle\theta \mid \cos \theta^{\prime}\right\rangle= & -\frac{i}{2} e^{i\left(\theta+\theta^{\prime}\right)} e^{i \theta^{\prime}} \Sigma_{n} e^{i m\left(\theta+\theta^{\prime}\right)} \\
& -\frac{i}{2} e^{i\left(\theta-\theta^{\prime}\right)}
\end{aligned}
$$

This function is so strongly peaked at $\theta= \pm \theta^{\prime}$ that the uncertainty in $\cos \hat{\phi}$ is zero. To show this we
calculate the expectation value $\langle\theta|\cos \hat{\phi}| \phi\rangle$.

$$
\begin{aligned}
\langle\theta|\cos \hat{\phi}| \theta\rangle & =\Sigma_{m_{n}}<m\left|\mathrm{e}^{-i m \theta} \cos \hat{\phi} \mathrm{e}^{i n \theta}\right| n>/ \Sigma_{m_{n}}<m\left|\mathrm{e}^{i(n-m) \theta}\right| n> \\
& =\frac{\Sigma_{n} \cos \theta-\mathrm{e}^{i \theta / 2}}{\Sigma_{n} 1}=\cos \theta
\end{aligned}
$$


The normalization swamps out the $e^{i \theta}$ term. Similarly $\langle\sin \hat{\phi}\rangle=\sin \theta$. The uncertainty in $\cos \phi \operatorname{can}$ be defined as $\langle\cos \hat{\phi}\rangle^{2}-\left\langle\cos ^{2} \phi\right\rangle$.

$$
\begin{aligned}
\left\langle\cos ^{2} \hat{\phi}>\right. & =\frac{1}{4} \frac{\left[\Sigma_{n}\left(e^{2 i \theta}+e^{-2 i \theta}+2\right)\right]+\text { finite terms }}{\Sigma_{n} 1} \\
& =\cos ^{2} \theta
\end{aligned}
$$

since the normalization again swamps out the finite terms. Hence

$$
\left\langle\cos ^{2}>-\langle\cos \rangle^{2}=0\right.
$$

In fact, one can show

$$
\left\langle\left(\cos \phi-\langle\cos \phi>)^{n}\right\rangle=0\right.
$$

If an oscillator is in a state $|\theta\rangle$ and $\sin \hat{\phi}$ or $\cos \hat{\phi}$ are measured, it is overwhelmingly probably that the measurements will yield $\cos \theta$ and $\sin \theta$.

Thus, although the states $|\theta\rangle$ are not eigenstates of a Hermitian $\hat{\phi}$, they are states for which the uncertainties in the non-commuting operators $\sin \hat{\phi}$ and $\cos \hat{\phi}$ are zero!

We can now see to what extent an oscillator can be used as a clock. We start by preparing the oscillator in the state

$$
\sum_{m} e^{i m \theta}|m>=| \theta>
$$

After time $t$ the state will have become

$$
|\theta-\omega t\rangle
$$

Since the uncertainties in $\sin \hat{\phi}$ and $\cos \hat{\phi}$ are "zero" in such states, simultaneous measurements of both can be performed to any degree of accuracy. That is, given any $\delta>0$ both sin $\hat{\phi}$ and $\cos \hat{\phi}$ can be measured within accuracy $\delta$. But from what was said before, it is overwhelmingly probably that the measurement will yield $\cos (\theta+\omega t)$ and $\sin (\theta+\omega t)$ within accuracy $\delta$ which uniquely tell us the value of $t$. And, in fact, any quantum oscillator can be used as an arbitrarily accurate clock if its $\cos \hat{\phi}$ and $\sin \hat{\phi} \operatorname{can}$ be measured.

\section{Periodic Systems}

Now why does the phase operator have such strange properties? Why can we not define the conjugate to the $\hat{n}$ operator? The reason is that the $\hat{n}$ operator does not have all integer eigenvalues but only positive values. To understand this better it is instructive to examine the conjugate to an operator with such a full integer spectrum from $-\infty$ to $+\infty$.

The system is the angle-angular momentum system which has many formal similarities with the phaseenergy system. Consider a bead on a circular wire. The position of the bead will be denoted by $\theta$, and the momentum conjugate to $\theta$ is $L$, the angular momentum. The allowable wave functions are functions of $\theta$ defined between $\theta=-\pi$ and $\theta=+\pi$. In order to handle the difficulties that arise at the endpoints of the interval the functions are usually continued past the ends periodically in $\theta$. Hence we consider the space of periodic functions of $\theta$, where $\theta$ can be considered the rotation parameter. Letting $\hat{L}=-i \frac{\partial}{\partial \theta}$ we get

$$
-i \frac{\partial}{\partial \theta} f_{m}=m f_{m} \quad \text { and } \quad f_{m}(\theta)=e^{i m \theta}
$$


and as usual $m$ must be an integer to insure the periodic character of $f_{m}(\theta)$.

The commutation relations for $\theta$ and $\hat{L}$ are usually taken to be

$$
[\hat{L}, \hat{\theta}]=i
$$

However, this is clearly inconsistent. Taking matrix elements

$$
\begin{aligned}
& \langle m|\hat{L} \hat{\theta}-\hat{\theta} \hat{L}| n\rangle=i \delta_{m n} \\
& (m-n)\langle m|\hat{\theta}| n\rangle=i \delta_{m n}
\end{aligned}
$$

which is impossible for $m=n$.

The difficulty lies in not treating the periodic nature of $\theta$ correctly. $\theta$ is taken to be the rotation parameter and as such takes on values from $-\infty$ to $+\infty$. Clearly $\theta$ operating on a periodic $f(\theta)$ gives a nonperiodic function. Hence multiplication by $\theta$ is not a good operator in the space of periodic functions. To remedy this we introduce an operator $\hat{\phi}$ which is periodic in $\theta$ :

$$
\hat{\phi} f(\theta)=\theta f(\theta), \quad-\pi<\theta<\pi
$$

and is periodic. The operator $\hat{\phi}$ is represented in Figure 1.

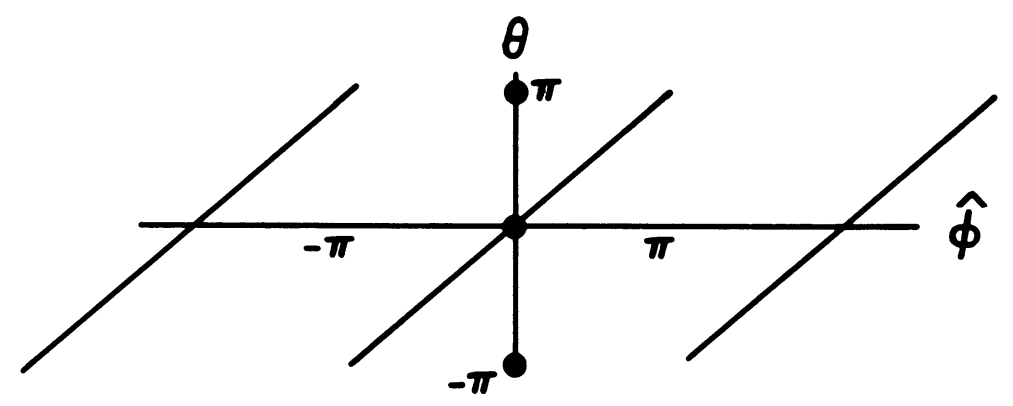

FIGURE 1

But $[\hat{L}, \hat{q}]$ is $i \frac{d \hat{q}}{d \theta}$ and not $i \frac{d \hat{q}}{d \hat{\phi}}$. In particular

$$
[\hat{L}, \hat{\phi}]=i \frac{d \hat{\phi}}{d \theta}=i(1-2 \pi \delta(\hat{\phi}-\pi))
$$

The choice of the delta function is somewhat arbitrary. It signifies that in a full $2 \pi$ rotation the value of $\phi$ must somewhere jump through $2 \pi$. We conventionally choose this point to be $\phi=\pi$. Evaluating this commutator assuming $\langle m \mid \theta\rangle=\frac{1}{2 \pi} e^{i m} \theta$, where $|\theta\rangle$ is an eigenvector of $\hat{\phi}$, we obtain

$$
\langle r|[\hat{L}, \hat{\phi}]| s\rangle=i \delta_{r s}-i e^{-i(r-s) \pi}
$$

This is the general relation between a momentum and periodic coordinates and one finds that any argument based on the $c$ number character of $[\hat{L}, \hat{\phi}]$ must be revised. Let us suppose there exist a pair of hermitian operators $\hat{L}$ and $\hat{\phi}$ such that $[\hat{L}, \hat{\phi}]=i(1-2 \pi \delta(\hat{\phi}-\pi))$,
$\hat{L}$ with a discrete integer spectrum and $\hat{\phi}$ with a continuous spectrum from $-\pi$ to $\pi$. Can we infer anything about the spectrum $\hat{L}$ ? To answer this we introduce an operator $\hat{\mathscr{L}}=-i \frac{d}{d \phi}$ with the restriction that $\hat{\mathscr{L}} f(\theta)=-i \frac{d f}{d \theta}$ if $f(\pi)=f(-\pi)$ and $\hat{\mathscr{L}} f(\theta)=-i \frac{d f}{d \theta}-i[f(-\pi)-f(\pi)] \delta(\theta-\pi)$ otherwise. Now one can 
prove that $[\hat{\mathscr{L}}, \hat{\phi}]=[\hat{L}, \hat{\phi}]$ and that $[\hat{\mathscr{L}}-\hat{L}, \hat{\phi}]=0$. This means that $\hat{L}=\hat{\mathscr{L}}+g(\hat{\phi})$ if $\hat{\phi}$ is to have a complete set of eigenvectors. The $g(\hat{\phi})$ can always be removed by unitary transformation, hence we lose no generality in assuming $\hat{L}=\hat{\mathscr{L}}$ [5]. But now the eigenstates of $\hat{\mathscr{L}}$ are clearly $\int d \theta \mathrm{e}^{i m} \theta \mid \theta>$ and its spectrum, $m$ goes from $-\infty$ to $+\infty$. That is to say if $\hat{L}$ and $\hat{\phi}$ obey the commutation rules of conjugate variables it is possible to show that there exist functions of $\theta$ which are eigenfunctions of $\hat{L}$ with all integer eigenvalues. Clearly angular momentum has the required spectrum.

This sheds light on the phase operator. We see that since $\hat{n}$ goes from 0 to $+\infty$ it is impossible to find any phase operator that will be periodic in time and have the properties required by a conjugate of $\hat{n}$. The point is that because there are no negative energy eigenvalues the phase operator can't exist. We have proved a generalization of this theorem for arbitrary systems. A time operator cannot exist for any system with a lowest energy state.

\section{Phase Difference of Two Oscillators}

To further understand the nature of phase and its relation to interferences, consider the $\cos \left(\hat{\phi}_{1}-\hat{\phi}_{2}\right)$ $\sin \left(\hat{\phi}_{1}-\hat{\phi}_{2}\right)$ operators for a pair of oscillators. We define them in a manner similar to the $\cos \hat{\phi}$ and $\sin \hat{\phi}$ for a single oscillator. Suppose $\hat{e}^{i \phi_{1}}, \hat{e}^{-i \phi_{1}}, \hat{e}^{i \phi_{2}}, \hat{e}^{-i \phi_{2}}$ are the exponential phase variables for the separate 1 and 2 oscillators. Then define $\hat{e}^{i\left(\phi_{1}-\phi_{2}\right)}=\hat{e}^{i \phi_{1}} \hat{e}^{-i \phi_{2}}$ (remember that they commute), $\hat{e}^{-i\left(\phi_{1}-\phi_{2}\right)}=$ $\hat{e}^{i \phi_{2}} \hat{e}^{-i \phi_{1}}$

$$
\begin{aligned}
2 \cos \left(\hat{\phi}_{1}-\hat{\phi}_{2}\right) & =\hat{e}^{i\left(\phi_{1}-\phi_{2}\right)}+\hat{e}^{-i\left(\phi_{1}-\phi_{2}\right)} \\
2 i \sin \left(\hat{\phi}_{1}-\hat{\phi}_{2}\right) & =\hat{e}^{i\left(\phi_{1}-\phi_{2}\right)}-\hat{e}^{-i\left(\phi_{1}-\phi_{2}\right)}
\end{aligned}
$$

The eigenvalue spectrum of $\cos \left(\hat{\phi}_{1}-\hat{\phi}_{2}\right)$ and $\sin \left(\hat{\phi}_{1}-\hat{\phi}_{2}\right)$ has some interesting properties. Let $\mid \psi>$ be an eigenstate of $\cos \left(\hat{\phi}_{1}-\hat{\phi}_{2}\right)$.

$$
\cos \left(\hat{\phi}_{1}-\phi_{2}\right)|\psi>=\lambda| \psi>
$$

Let $\left.|\psi\rangle=\sum_{m n} a_{m n}|m\rangle \mid n\right)$ where $|m\rangle$ is a number eigenstate of oscillator 1 and $\mid n$ ), a state of oscillator

2. Then

$$
\begin{aligned}
2 \cos \left(\hat{\phi}_{1}-\hat{\phi}_{2}\right) \mid \psi>= & \left.\sum_{m, n=1}^{\infty}\left(a_{m-1, n+1}+a_{m+1, n-1}\right)|m>| n\right) \\
& \left.\left.+\sum_{m=1}^{\infty} a_{m-1,1}|m>| 0\right)+\sum_{n=1}^{\infty} a_{1, n-1}|0>| n\right) \\
= & \lambda \sum_{m n} a_{m n}|m>| n>
\end{aligned}
$$

which gives

$$
\begin{aligned}
a_{m-1, n+1}+a_{m+1, n-1} & =\lambda a_{m n} \\
a_{m-1,1} & =\lambda a_{m, 0} \\
a_{1, n-1} & =\lambda a_{0, n}
\end{aligned}
$$

Notice that each term of each equation is an amplitude for a total number of excitations $n+m$. This means that we may separately write the recursion relations involving a total of $R$ excitations. Let $R$ be the total 
number of excitations and $m$, the excitation in oscillator 1 . Denote the amplitude $a_{n, R-m}$ by $R_{m}$. Then

$$
\begin{aligned}
R_{m-1}+R_{m+1} & =\lambda R_{m} \quad \text { for } \quad m \neq 0, R \\
R_{R-1} & =\lambda R_{R} \\
R_{1} & =\lambda R_{0}
\end{aligned}
$$

Each eigenstate of $\cos \left(\hat{\phi}_{1}-\hat{\phi}_{2}\right)$ determined by these equations is an eigenstate of $m+n$. This is simply a consequence of the commutation relation

$$
\left[\cos \left(\hat{\phi}_{1}-\hat{\phi}_{2}\right), \hat{n}_{1}+\hat{n}_{2}\right]=0
$$

As we have seen, the general solution to the first of equations (41) is $R_{n}=a p^{n}+b p^{-n}$ where $\lambda=p+\frac{1}{p}$. The second 2 relations determine the allowable values of $p$. The values of $p$ are plotted on a complex plane in Figure 2. Note that the state with $p=1 / p^{\prime}$ is the same as the state with $p=p^{\prime}$. Hence only $1 / 2$ the

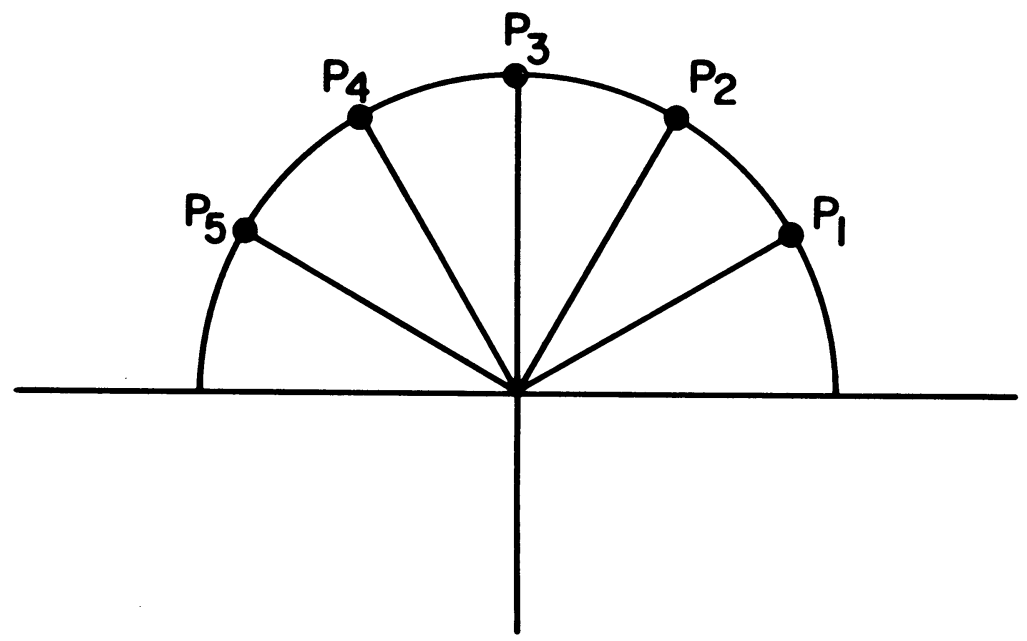

FIGURE 2

plane need be used. We shall use the top half. Also, the states with $p= \pm 1$ do not exist. Altogether then there are $R+1$ orthonormal eigenstates of $\cos \left(\hat{\phi}_{1}-\hat{\phi}_{2}\right)$, all of which are eigenstates of $\hat{m}+\hat{n}$. But there are $R+1$ linearly independent eigenstates of $\hat{m}+\hat{n}$ and hence the eigenstates of $\cos \left(\hat{\phi}_{1}-\hat{\phi}_{2}\right)$ form a complete set for the Hilbert space of $R$ excitations. Considering the total of all eigenstates of $\cos \left(\hat{\phi}_{1}-\hat{\phi}_{2}\right)$ of this type, they form a complete set and $\cos \left(\hat{\phi}_{1}-\hat{\phi}_{2}\right)$ can have no other eigenstates or
eigenvalues.

The eigenvalues of $\cos \left(\hat{\phi}_{1}-\hat{\phi}_{2}\right)^{6}$ do not include all the points from -1 to +1 but only a countable infinity of them, namely $\cos \theta$, where $\theta$ is a rational multiple of $\pi$. We do not expect the phase difference variables to have many significant analogies with classical phase variables for states of low excitation since the eigenvalue spectrum is very unlike the classically allowed values of $\cos \left(\theta_{1}-\theta_{2}\right)$ and $\sin \left(\theta_{1}-\theta_{2}\right)$. However, let us look at states with a large number of quanta for which the cos, sin spectrum

Consider the "states with phase difference $\theta$ "

$$
\left|R, \theta>=\sum_{0}^{R} e^{i m \theta}\right| m>\mid R-m>
$$

and the amplitudes 


$$
<\cos \theta^{\prime}, R \mid R, \theta>=\mathrm{e}^{i \theta^{\prime}} \sum_{0}^{R} \mathrm{e}^{i n\left(\theta-\theta^{\prime}\right)}+\mathrm{e}^{-i \theta^{\prime}} \sum_{0}^{R} \mathrm{e}^{i n\left(\theta-\theta^{\prime}\right)}
$$

Now if $R$ is large, this function is very strongly peaked at $\theta=\theta^{\prime}$ and $\theta=-\theta^{\prime}$ or equivalently $\cos \theta=$ $\cos \theta^{\prime}$. Similarly for $\sin \hat{\phi}$.

The expectation value of $\cos \hat{\phi}$ in state $|R, \theta\rangle$ is $\frac{R}{R+1} \cos \theta$ and

$$
<\cos \hat{\phi}\rangle^{2}=\frac{R^{2}}{(R+1)^{2}} \cos ^{2} \theta
$$

Also

$$
\left\langle\cos ^{2} \hat{\phi}>=\frac{R-1}{R+1} \cos ^{2} \theta\right.
$$

and the uncertainty in $\cos \hat{\phi}$ is

$$
\left\langle\cos ^{2}>-<\cos >^{2}=\frac{1-2 R}{(R+1)^{2}} \cos ^{2} \theta\right.
$$

which goes as $1 / R$ for large $R$. This justifies our use of $|R, \theta\rangle$ as states of well known phase difference when $R$ is large.

\section{Uncertainty Relations}

The primary reason for Bohm and Aharonov's investigation of time operators was to demonstrate the energy-time uncertainty relations. We have shown that an oscillator's phase can be used as a clock variable, but we have not investigated limitations on the clock's accuracy due to the uncertainty principle. What is a good measure of the uncertainty in the phase? We should like to find some measure of probability for phase which reflects the probability distribution for its measurable trigonometric functions, and use this to define the uncertainty relations. Now the phase must be measured by measuring its trigonometric functions. Suppose our apparatus is designed to measure the quantity $\cos (\hat{\phi}+\epsilon)$. Denote the probability that the measurement yield $\cos (\hat{\phi}+\epsilon)=\cos (\theta+\epsilon)$ by $P[\cos (\hat{\phi}+\epsilon)=\cos (\theta+\epsilon)]$. Then classically

$$
P[\cos (\hat{\phi}+\epsilon)=\cos (\theta+\epsilon)]=P[\hat{\phi}=\theta]+P[\hat{\phi}=-\theta-2 \epsilon]
$$

where $P[\hat{\phi}=\theta]$ is the probability that $\hat{\phi}$ has value $\theta$.

Another way of saying the same thing is that if the original state is displaced through phase angle $\epsilon$ then the probability, $P \epsilon$, that in the new state $\cos \hat{\phi}=\cos (\theta+\epsilon)$ is the probability, $P_{0}$, that in the old state $\hat{\phi}=\theta$ plus the probability that in the old state $\hat{\phi}=-\theta-2 \epsilon$.

$$
P \epsilon[\cos \hat{\phi}=\cos (\theta+\epsilon)]=P_{0}(\hat{\phi}=\theta)+P_{0}(\hat{\phi}=-\theta-2 \epsilon)
$$

Now quantum mechanically a measurement of $\cos \hat{\phi}$ does not distinguish between $+\phi$ and $-\phi$. Because of this we must replace probabilities by corresponding probability amplitudes.

$$
e^{i \epsilon} A \epsilon[\cos \hat{\phi}=\cos (\theta+\epsilon)]=A_{0}(\hat{\phi}=\theta)+e^{i \alpha} A_{0}(\hat{\phi}=-\theta-2 \epsilon)
$$

where $\mathrm{e}^{i \epsilon}$ compensates for the rotation through angle $\epsilon$ and $\mathrm{e}^{i a}$ is an arbitrary undetermined phase. This is the equation we will use to define $A_{0}(\hat{\phi}=\theta)$. This definition is not trivial because for different values of $\epsilon$ the measurements of $\cos \hat{\phi}$ are not compatible and it is not clear, a priori, that a function $A_{0}(\hat{\phi}=\theta)$ can be found which obeys (48) independently of the value of $\epsilon$. 
Suppose now that equation (48) does define an amplitude $A_{0}$ for the phase taking on various values. Then we can be sure that for any value of $\epsilon$ the projections of a state onto an eigenstate of $\cos (\hat{\phi}+\epsilon)$ with eigenvalue $\cos (\theta+\epsilon)$ will be large only if either $A_{0}(\hat{\phi}=\theta)$ or $A_{0}(\hat{\phi}=-\theta-2 \epsilon)$ is large. Furthermore, suppose $A_{0}(\hat{\phi}=\theta)$ is large. For any given $\epsilon, A \epsilon(\cos \hat{\phi}=\cos (\theta+\epsilon))$ may not be large. But we can always find some value of $\epsilon$ for which it will be large. For example, suppose $A_{0}\left(\hat{\phi}=\theta^{\prime}\right)$ is small. Then let $\epsilon=-\left(\theta-\theta^{\prime}\right) / 2$. Thus if $\cos (\hat{\phi}+\epsilon)$ is measured in a state in which it is probable that it equals a given value, then one of the two angles which could yield this value must be in the region where $A(\hat{\phi}=\theta)$ is large. Also, for some method of measuring the phase (measuring $\cos (\hat{\phi}+\epsilon)$ the probability will be large that $\cos (\theta+\epsilon)$ is obtained for every angle in this region. We conclude that the width of an $A$ function obeying (48) is a true measure of the uncertainty in phase, taking into account both the ordinary quantum mechanical uncertainty in measuring the trigonometric functions of phase and the incompatibility of the different methods of measuring it. Now it remains to construct a suitable $A$.

Consider any state $|\psi\rangle$ which can be expanded in the form

$$
\sum_{n} \int d \bar{\theta} \rho(\bar{\theta}) e^{i m \bar{\theta}} \mid m>
$$

such that the Fourier expansion of $\rho(\bar{\theta})$ contains only exponentials with negative integers multiplying $\bar{\theta}$.

$$
\rho(\bar{\theta})=\sum_{n} a_{m} \mathrm{e}^{-i m \bar{\theta}}
$$

To find $A \epsilon[\cos \hat{\phi}=\cos (\theta+\epsilon)]$ we rotate $\mid \psi>$ through angle $\epsilon$ and take its projection on cos states

$$
\begin{aligned}
A \epsilon[\cos \hat{\phi}=\cos (\theta+\epsilon)]=\left\langle\cos (\theta+\epsilon)\left|\sum_{m} \int d \bar{\theta} \rho(\bar{\theta}) \mathrm{e}^{i m \bar{\theta}} \mathrm{e}^{i m \epsilon}\right| m\right\rangle & \\
& =\mathrm{e}^{i(\theta+\epsilon)} \sum_{m} \mathrm{e}^{i m(\theta+2 \epsilon)} a_{m}-\mathrm{e}^{-i(\theta+\epsilon)} \sum_{m} \mathrm{e}^{-i m \theta} a_{m}
\end{aligned}
$$

Now since $\rho$ has Fourier projections only on negative integer exponentials we can write this as

$$
\mathrm{e}^{i(\theta+\epsilon)} \rho(-\theta-2 \epsilon)-\mathrm{e}^{-i(\theta+\epsilon)} \rho(\theta)=\mathrm{e}^{-i \epsilon}\left[\mathrm{e}^{-i(-\theta-2 \epsilon)} \rho(-\theta-2 \epsilon)-\mathrm{e}^{-i \theta} \rho(\theta)\right)
$$

But this is the same form as equation (48) with $A(\hat{\phi}=\theta)=e^{-i \theta} \rho(\theta)$. The factor $e^{-i \theta}$ can be absorbed into the relative phase factor $e^{i a}$. Actually the particular choice of phase can be made plausible by a selfconsistency argument but, since our only use of $A$ will be to define an uncertainty for $\phi$, the question of the
relative phase in unimportant. Thus for such

To show that

the $\mid \theta>$ states. Although they are expanded in the manner described, we make use of a special property of

$$
\int \mid \theta>\langle\theta| d \theta=I
$$

Any state can be expanded in $|\theta\rangle$.

$$
|\psi\rangle=\int d \theta\langle\theta \mid \psi\rangle|\theta\rangle
$$
The over completeness of the $\mid \theta>$ means that $\mid \psi>$ may be expanded with many weighting factors other than
$\langle\theta \mid \psi\rangle$ but only $\langle\theta| \psi>$ has projections only on the negative integer exponents. 


$$
\langle\theta \mid \psi\rangle=\sum_{m} e^{-i m \theta}\langle m \mid \psi\rangle
$$

Also, if

$$
\int[\langle\theta \mid \psi\rangle+f(\theta)]|\theta\rangle=|\psi\rangle
$$

then

$$
\int f(\theta) \mid \theta>=0
$$

But then

$$
\sum_{m} \int d \theta f(\theta) e^{i m \theta} \mid m>=0
$$

The $|m\rangle$ are linearly independent so that

$$
\int d \theta f(\theta) e^{i m \theta}=0
$$

And $f$ is totally composed of positive integer exponentials. Hence for any state $|\psi\rangle$ we may simply choose $A$ to be $\langle\theta \mid \psi\rangle$.

The uncertainty relations become statements about the widths of functions

$$
\rho(\theta)=\sum_{0}^{\infty} e^{-i m \theta} a_{m}
$$

with $a_{m}$ being spread over $\Delta_{m}$. Such functions have the property that their width, $\Delta \theta$ times $\Delta_{m}$, is greater than 1 when $\Delta \theta$ becomes small. As the spread in $m$ becomes small, $\Delta \theta$ must increase until $\rho(\theta)$ is spread over $2 \pi$. More exact statements could be derived, but our purpose here is only to show such uncertainty relations can be defined by the rigorous use of well defined quantum operator trigonometric functions of phase.

\section{References}

1. D. BOHM and Y. AHARONOV, Phys. Rev. 122, 1649 (1961).

2. W. HEITLER, Quantum Theory of Radiation Chap. II. Oxford University Press (1954).

3. P. DIRAC, Quantum Theory of Emission and Absorption in Quantum Electrodynamics (Edited by J. SCHWINGER). Dover Publications, New York (1958).

4. Actually this function is $\delta\left(\theta \cdot \theta^{\prime}\right)+i \cot 1 / 2\left(\theta-\theta^{\prime}\right)$.

5. This argument is the same as the one used by Dirac for momentum and position. See P. DIRAC, The Principles of Quantum Mechanics (4th ed.) Chap. 4. Oxford University Press (1958).

6. $\cos \hat{\phi}$ and $\theta$ refer to the phase difference of the two oscillators for the rest of this section. 